\title{
Differential Renormalization-Group Approach to the Layered sine-Gordon Model
}

\author{
I. Nándor** \\ Institute of Nuclear Research of the Hungarian Academy of Sciences, \\ H-4001 Debrecen, P.O.Box 51 Hungary; \\ Max Planck Institute for Nuclear Physics, \\ P.O.Box 103980, Heidelberg 69029, Germany \\ K. Sailer \\ Department of Theoretical Physics, University of Debrecen, \\ H-4010 Debrecen, P.O.Box 5 Hungary
}

(Dated: November 15, 2018)

\begin{abstract}
New qualitative picture of vortex length-scale dependence has been found in recent electrical transport measurements performed on strongly anisotropic $\mathrm{Bi}_{2} \mathrm{Sr}_{2} \mathrm{CaCu}_{2} \mathrm{O}_{8}$ single crystals in zero magnetic field. This indicates the need for a better description of the $3 \mathrm{D} / 2 \mathrm{D}$ crossover in vortex dimensionality. The vortex-dominated properties of high transition temperature superconductors with extremely high anisotropy (layered systems) are reasonably well described in the framework of the layered XY model which can be mapped onto the layered sine-Gordon model. For the latter we derive an exact renormalization group (RG) equation using Wegner's and Houghton's approach in the local potential approximation. The agreement of the UV scaling laws find by us by linearizing the RG equations with those obtained previously in the literature in the dilute gas approximation makes the improvement appearant which can be achieved by solving our RG equations numerically.
\end{abstract}

PACS numbers: Valid PACS appear here

Keywords: Renormalization group method, layered sine-Gordon model

*Electronic address: nandori@atomki.hu 


\section{INTRODUCTION}

Vortex dominated properties of high-temperature superconductors (HTSC) are usually investigated by means of electrical transport measurements which have raised many questions about the critical behaviour and the length-scale dependence of vortices in layered systems. The HTSC materials have a layered structure and usually consist of copper-oxide superconducting planes separated by insulators. In case of extremely high anisotropy like in $\mathrm{Bi}_{2} \mathrm{Sr}_{2} \mathrm{CaCu}_{2} \mathrm{O}_{8}$ (BSCCO) single crystals, the coupling between the $\mathrm{CuO}_{2}$ planes is weak. Due to this weak coupling, one of the commonly accepted models is the layered XY model (Pierson and Valls 1992) where the weak interlayer coupling is given by a Lawrence-Doniach type term (Pierson et. al. 1992). The topological excitations in superconducting layers are vortex-antivortex pairs. Two such pairs belonging to neighbouring layers can form vortex loops due to the Josephson coupling.

The layered XY model can be mapped onto the layered sine-Gordon (LSG) model (Pierson and Valls 1994, 1992) or it can be transformed into the layered Coulomb gas (LCG) model (Pierson 1995). The critical behaviour of vortices and vortex loops in the LSG and LCG models have been investigated by renormalization group (RG) methods using the dilute gas approximation (Pierson 1995, Pierson et. al. 1992) which assumes low vortex fugacity. However, the vortex fugacity becomes large above the critical temperature (but below the Ginzburg-Landau transition temperature) when individual vortices can be spontaneously created although the sample is still in the superconducting phase.

Recent current transport measurements (Vad et al. 2004) performed on BSCCO superconducting single crystals show a new qualitative picture of vortex behaviour above the critical temperature (Nandori et. al. 2004). Using a multi-contact 'DC flux transformer' configuration, the temperature dependence of primary and secondary voltages are measured in zero magnetic field. (The side of the crystal where the current is injected is called the primary one, its opposite side is the secondary one.) According to the theoretical predictions based on the dilute gas approximation (Pierson 1995), above the critical temperature one should expect the decrease of the secondary voltage to zero as a function of the temperature. Instead, the experimental data reveal a new feature (Nandori et. al. 2004), a second peak appears in the temperature dependence of the secondary voltage. A possible reason for the disagreement between the theory and experiment is the usage of the dilute gas approxi- 
mation. This indicates the need for a better description of length-scale dependence of the vortex dimensionality at the $3 \mathrm{D} / 2 \mathrm{D}$ crossover and hence the modification of the $\mathrm{RG}$ analysis (Nandori et. al. 2004).

The purpose of this paper is to propose a theoretical study of the LSG model using the differential RG approach (Wilson and Kogut 1974, Wilson 1983) in order to improve the treatment of the length-scale dependence of vortices. In section 2 , the definition of the layered models and their interrelations are summarized. After giving a short sketch of Wegner's and Houghton's RG method (Wegner and Houghton 1973) for models with two interacting scalar fields in section 3, we apply it to the LSG model using the local potential approximation (Polonvi 2001) in section 4. The UV scaling laws are found and discussed in section 5 on the base of the linearized RG equations. The comparison of the UV scaling laws to those obtained previously in Refs. (Pierson and Valls 1992, Pierson et. al. 1992) using a momentum-space RG method with smooth cut-off in the dilute gas approximation is also given, and the possibility of an improved treatment of the RG flow by our method is pointed out. Finally the results are summarized in section 6 .

\section{LAYERED MODELS}

The phase structure of HTSC materials is usually investigated in the framework of the Ginzburg-Landau (GL) theory of superconductivity at the phenomenological level. The GL theory is based on a variational method which has been applied to the gradient expansion of the free-energy in powers of the complex order parameter $\psi$ and its derivatives $\nabla \psi$ where $|\psi|^{2}$ represents the local density of superconducting electron pairs. In case of strong anisotropy and in the absence of external fields the free energy becomes (Nandori et. al. 2004)

$$
F=\sum_{n} \int \mathrm{d}^{2} r\left(\frac{1}{2} J_{\|}\left(\nabla \phi_{n}\right)^{2}+J_{\perp}\left[1-\cos \left(\phi_{n}-\phi_{n-1}\right)\right]\right)
$$

where $\psi_{n}(r)=\psi_{0} \exp \left[i \phi_{n}(r)\right]$ is assumed with $\psi_{0}$ real and identical in every layer $n$. The coupling constants of the model are $J_{\|}=\hbar^{2} \psi_{0}^{2} / m_{a b}$ and $J_{\perp}=\hbar^{2} \psi_{0}^{2} / m_{c} s^{2}$ with the effective masses $m_{a b}, m_{c}$, and the interlayer distance $s$. The last term is related to the LawrenceDoniach term, in which the coupling between the layers is the Josephson coupling. Expanding the cosine term in Taylor series around zero, one arrives at the layered two-dimensional 
XY model (Pierson and Valls 1992):

$$
H_{L X Y}=\frac{1}{2} \sum_{n} \int \mathrm{d}^{2} r J_{\|}\left(\nabla \phi_{n}\right)^{2}+\frac{1}{2} \sum_{n} \int \mathrm{d}^{2} r J_{\perp}\left(\phi_{n}-\phi_{n-1}\right)^{2} .
$$

The layered XY model can be mapped onto the LSG or LCG models (Pierson and Valls 1994, Pierson et. al. 1992). For a system consisting of two layers the Lagrangian of the LSG model reads as (Pierson and Valls 1992)

$$
L_{L S G}=\frac{1}{2}\left[\left(\partial \phi_{1}\right)^{2}+\left(\partial \phi_{2}\right)^{2}\right]+\frac{1}{2} \tilde{J}\left(\phi_{1}-\phi_{2}\right)^{2}+\tilde{u}\left[\cos \left(\beta \phi_{1}\right)+\cos \left(\beta \phi_{2}\right)\right],
$$

where $\phi_{1}, \phi_{2}$ are one-component scalar fields, $\tilde{u}$ is the fugacity parameter for the vortices of the model, $\beta^{2}=J_{\|}$and $\tilde{J}=J_{\perp} /\left(\Lambda^{2} J_{\|}\right)$are dimensionless coupling constants and $\Lambda$ stands for the UV momentum cutoff. The second term on the r.h.s. describes the interaction of the layers. In Refs. (Pierson and Valls 1992, Pierson et. al. 1992) RG flow equations,

$$
k \frac{d}{d k} \beta^{2}=\frac{1}{4 \pi}\left(\tilde{u}^{2} \beta^{4}-4.5 \beta^{4} \tilde{u}^{2} \tilde{J} \ln \tilde{J}\right), \quad k \frac{d}{d k} \tilde{u}=\tilde{u}\left(\frac{\beta^{2}}{4 \pi}-2\right), \quad k \frac{d}{d k} \tilde{J}=-2 \tilde{J},
$$

where $k$ is the momentum scale, have been obtained for the coupling constants of the LSG model (3) with the help of a momentum space RG analysis using a smooth (anisotropic) momentum cutoff and the dilute gas approximation. The latter assumes that the fugacity of the vortex gas, i.e. the coupling constant $\tilde{u}$ in (3) is small.

\section{DIFFERENTIAL RG APPROACH}

The renormalization of the LSG model is presented in this section by means of the differential RG approach performed in momentum space with sharp cutoff (Polonvi 2001). The blocking transformations (Wilson and Kogut 1974, Wilson 1983) are realized by successive elimination of the field fluctuations according to their decreasing momentum in infinitesimal steps. We use Wegner's and Houghton's method (WH-RG) (Wegner and Houghton 1973) when the high-frequency modes are integrated out above the moving sharp momentum cutoff $k$ and the physical effects of the eliminated modes are built in the scale-dependence of the coupling constants.

Assuming that the generating functional does not change under the infinitesimal blocking transformation when the momenta of the eliminated modes are taken from a thin momentum shell with radius $k$ and thickness $\Delta k$, the blocking relation

$$
e^{-S_{k-\Delta k}[\phi]}=\int \mathcal{D}\left[\phi^{\prime}\right] e^{-S_{k}\left[\phi+\phi^{\prime}\right]},
$$


is obtained for the blocked action $S_{k}[\phi]$ (Polonvi 2001). The field variables $\phi$ and $\phi^{\prime}$ contain Fourier modes with momenta $p<k-\Delta k$ and $k-\Delta k<p<k$, respectively. Expanding the action $S_{k}\left[\phi+\phi^{\prime}\right]$ in Taylor series around its saddle point, the path integral can be evaluated and the WH-RG method (Wegner and Houghton 1973) provides the functional RG equation

$$
S_{k-\Delta k}[\phi]=S_{k}[\phi]+\frac{\hbar}{2} \operatorname{tr} \ln \left(S_{k}^{\prime \prime}[\phi]\right)+O\left(\hbar^{2}\right),
$$

where $S_{k}^{\prime \prime}$ denotes the second functional derivative of the blocked action with respect to the field and the trace is over the momentum shell $[k-\Delta k, k]$. In the limit $\Delta k \rightarrow 0$ Eq. (66) reduces to an exact integro-differential equation for the functional $S_{k}[\phi]$ because the small parameter $\Delta k / k$ suppresses the higher-loop contributions (Polonvi 2001). The generalization of Eq.(6) for two, interacting scalar fields is straightforward following the method proposed in (Liao and Polonvi 1995) and results in

$$
S_{k-\Delta k}\left[\phi_{1}, \phi_{2}\right]=S_{k}\left[\phi_{1}, \phi_{2}\right]+\frac{\hbar}{2} \operatorname{tr} \ln \left(S_{k}^{11}\left[\phi_{1}, \phi_{2}\right] S_{k}^{22}\left[\phi_{1}, \phi_{2}\right]-S_{k}^{12}\left[\phi_{1}, \phi_{2}\right] S_{k}^{21}\left[\phi_{1}, \phi_{2}\right]\right),
$$

where $S_{k}^{i j}\left[\phi_{1}, \phi_{2}\right]$ denote the second functional derivatives of the blocked action with respect to $\phi_{i}$ and $\phi_{j}$.

If the saddle point is non-trivial in (5), a spinodal instability occurs at some critical momentum scale $k_{c}$, and the WH-RG equation (6) loses its validity (Alexandre and Polonvi 1999). Then, the blocking produces a tree-level effect (Alexandre and Polonvi 1999). In case of two interacting scalar fields the tree-level blocking relation reads as

$$
S_{k-\Delta k}\left[\phi_{1}, \phi_{2}\right]=\min _{\phi_{1}^{c l}, \phi_{2}^{c l}}\left(S_{k}\left[\phi_{1}+\phi_{1}^{c l}, \phi_{2}+\phi_{2}^{c l}\right]\right)
$$

where the minimum is sought over field configurations including only Fourier-modes with momenta from the infinitesimal momentum shell $k-\Delta k<p<k$.

\section{RG APPROACH TO THE GENERALIZED LSG MODEL}

In this section we specify the WH-RG equation (7) and the tree-level blocking relation (8) for the LSG model. First, the WH-RG equation and the tree-level blocking relation, have to be projected into a particular functional subspace, in order to reduce the search for a functional (the blocked action) to that of functions. Here we assume that the blocked action

contains only local interactions. Expanding it in powers of the gradients of the fields $\phi_{1}$ and 
$\phi_{2}$ and keeping only the leading order terms one arrives at the local potential approximation (LPA) (Polonvi 2001). In LPA the ansatz for the blocked action for two scalar fields can be written as

$$
S_{k}\left[\phi_{1}, \phi_{2}\right]=\int \mathrm{d}^{d} x\left[\frac{1}{2}\left(\partial_{\mu} \phi_{1}\right)^{2}+\frac{1}{2}\left(\partial_{\mu} \phi_{2}\right)^{2}+V_{k}\left(\phi_{1}, \phi_{2}\right)\right]
$$

with the blocked potential $V_{k}\left(\phi_{1}, \phi_{2}\right)$ satisfying the WH-RG equation

$$
k \partial_{k} V_{k}\left(\phi_{1}, \phi_{2}\right)=-k^{d} \alpha_{d} \ln \left(\frac{\left[k^{2}+V_{k}^{11}\right]\left[k^{2}+V_{k}^{22}\right]-\left[V_{k}^{12}\right]\left[V_{k}^{21}\right]}{k^{4}}\right)
$$

with $\alpha_{d}=\frac{1}{2} \Omega_{d}(2 \pi)^{-d}$, where $\Omega_{d}$ denotes the solid angle in $d$ dimensions and $V_{k}^{i j}=$ $\partial_{\phi_{i}} \partial_{\phi_{j}} V_{k}\left(\phi_{1}, \phi_{2}\right)$. In order to remove the trivial scale-dependence of the coupling constants, the WH-RG equation (10) has to be rewritten in terms of dimensionless quantities. For dimensions $d=2$, the dimensionless form of Eq.(10) reads as

$$
\left(2+k \partial_{k}\right) \tilde{V}_{k}\left(\phi_{1}, \phi_{2}\right)=-\alpha_{2} \ln \left(\left[1+\tilde{V}_{k}^{11}\right]\left[1+\tilde{V}_{k}^{22}\right]-\tilde{V}_{k}^{12} \tilde{V}_{k}^{21}\right)
$$

where $\tilde{V}_{k}\left(\phi_{1}, \phi_{2}\right)=k^{-2} V_{k}\left(\phi_{1}, \phi_{2}\right)$ has been introduced.

The argument of the logarithm in Eqs. (10) and (11) must be positive. If the argument vanishes or if it changes sign at a critical value $k_{\mathrm{c}}$, the WH equation (10) loses its validity for $k<k_{\mathrm{c}}$. This is a consequence of the spinodal instability, i.e. that of the vanishing of the restoring force for field fluctuations of momenta from the infinitesimal momentum shell. Such fluctuations can grow to those of finite amplitude and result in a non-vanishing sadlle-point configuration. Below the critical scale $k<k_{c}$ the tree-level blocking relation (8) can be used to find the saddle point. Looking for it among the configurations $\phi_{1}^{c l}$ and $\phi_{2}^{c l}$ having the form of plane waves one finds for the blocked local potential

$$
V_{k-\Delta k}\left(\phi_{1}, \phi_{2}\right)=\min _{\rho_{k}}\left[2 \rho_{k}^{2} k^{2}+\frac{1}{2} \int_{-1}^{1} \mathrm{~d} p V_{k}\left(\phi_{1}+2 \rho_{k} \cos (\pi p), \phi_{2}+2 \rho_{k} \cos (\pi p)\right)\right],
$$

where $\rho_{k}$ is the amplitude of the plane waves (Alexandre and Polonvi 1999). Notice that the layers in the LSG model are physically equivalent therefore the potential is invariant under the transformation $\phi_{1} \leftrightarrow \phi_{2}$ which implies identical plane-wave forms of $\phi_{1}^{c l}$ and $\phi_{2}^{c l}$ with the same amplitude $\rho_{k}$ and wave vector in the saddle point. For dimensions $d=2$, the tree-level relation (12) for dimensionless quantities reads as

$$
\tilde{V}_{k-\Delta k}\left(\phi_{1}, \phi_{2}\right)=\min _{\rho_{k}}\left[2 \rho_{k}^{2}+\frac{1}{2} \int_{-1}^{1} \mathrm{~d} p \tilde{V}_{k}\left(\phi_{1}+2 \rho_{k} \cos (\pi p), \phi_{2}+2 \rho_{k} \cos (\pi p)\right)\right] .
$$


As to the next, we specify the WH-RG equation (111) and the tree-level blocking relation (13) for the LSG model. On the one hand, the ansatz for the blocked potential should be reach enough in order to ensure that the RG flow does not leave the chosen subspace of blocked potentials. On the other hand, the ansatz for the blocked potential should preserve all symmetries of the original model at the UV cutoff scale $k=\Lambda$. In particular, the blocked potential for the LSG model should be invariant under the exchange of the field variables, $\phi_{1} \leftrightarrow \phi_{2}$ because the layers are physically equivalent and also reveal the symmetries $\phi_{i} \rightarrow-\phi_{i}$ and $\phi_{i} \rightarrow \phi_{i}+\frac{2 \pi}{\beta^{2}}$ (c.f. Eq. (3) ). Therefore, we choose the ansatz

$$
\tilde{V}_{k}\left(\phi_{1}, \phi_{2}\right)=\frac{1}{2} \tilde{J}_{k}\left(\phi_{1}-\phi_{2}\right)^{2}+\tilde{U}_{k}\left(\phi_{1}, \phi_{2}\right)
$$

where $\tilde{U}_{k}\left(\phi_{1}, \phi_{2}\right)$ is an arbitrary periodic function of the fields

$$
\tilde{U}_{k}\left(\phi_{1}, \phi_{2}\right)=\sum_{n, m}\left[\tilde{u}_{n m}(k) \cos \left(n \beta \phi_{1}\right) \cos \left(m \beta \phi_{2}\right)+\tilde{v}_{n m}(k) \sin \left(n \beta \phi_{1}\right) \sin \left(m \beta \phi_{2}\right)\right]
$$

with symmetric Fourier amplitudes $\tilde{u}_{n m}=\tilde{u}_{m n}, \tilde{v}_{n m}=\tilde{v}_{m n}$. The potential (15) defines the generalized LSG model which contains higher harmonics of the field variables. Notice, that the scale-dependence is entirely encoded in the coupling constants $\tilde{J}_{k}, \tilde{u}_{n m}(k)$ and $\tilde{v}_{n m}(k)$. Inserting this ansatz into the WH-RG equation (11),

$$
\begin{aligned}
& \left(2+k \partial_{k}\right)\left(\frac{1}{2} \tilde{J}_{k}\left(\phi_{1}-\phi_{2}\right)^{2}+\tilde{U}_{k}\left(\phi_{1}, \phi_{2}\right)\right)= \\
& -\alpha_{2} \ln \left(1+2 \tilde{J}_{k}+\tilde{U}_{k}^{11}+\tilde{U}_{k}^{22}+\tilde{J}_{k}\left(\tilde{U}_{k}^{11}+\tilde{U}_{k}^{22}+\tilde{U}_{k}^{12}\right)+\tilde{U}_{k}^{11} \tilde{U}_{k}^{22}-\left(\tilde{U}_{k}^{12}\right)^{2}\right),
\end{aligned}
$$

and separating the periodic and non-periodic parts, one arrives at the RG flow equations

$$
\begin{aligned}
\left(2+k \partial_{k}\right) \tilde{J}_{k}= & 0 \\
\left(2+k \partial_{k}\right) \tilde{U}_{k}\left(\phi_{1}, \phi_{2}\right)= & -\alpha_{2} \ln \left(1+2 \tilde{J}_{k}+\tilde{U}_{k}^{11}+\tilde{U}_{k}^{22}+\tilde{J}_{k}\left(\tilde{U}_{k}^{11}+\tilde{U}_{k}^{22}+\tilde{U}_{k}^{12}\right)\right. \\
& \left.+\tilde{U}_{k}^{11} \tilde{U}_{k}^{22}-\left(\tilde{U}_{k}^{12}\right)^{2}\right)
\end{aligned}
$$

since the argument of the logarithm is a periodic function of the fields. Notice that the dimensionless coupling constant $\tilde{J}_{k}$ has only a trivial tree-level evolution and hence the dimensionful $J=k^{2} \tilde{J}_{k}$ remains constant during the RG procedure. It is also important to note that the second equation of (17) keeps the periodicity with the same length of period, therefore $\beta$ has no scale-dependence in the LPA. 
In order to specify the tree-level blocking for the generalized LSG model, one has to insert the ansatz (14) and (15) into the tree-level relation (13). For the sake of simplicity we turn back to dimensionful parameters and the relation (12). Performing the integral and separating the periodic and the non-periodic parts, Eq. (12) reduces to two relations. One of them,

$$
J_{k-\Delta k}=J_{k},
$$

says that the dimensionful coupling constant $J$ remains unchanged under the tree-level blocking, so that the corresponding dimensionless coupling keeps its trivial scaling $\tilde{J}_{k}=J k^{-2}$ in LPA even below the critical scale $k_{c}$. The relation for the periodic part is

$$
\begin{aligned}
& U_{k-\Delta k}\left(\phi_{1}, \phi_{2}\right)=\min _{\rho_{k}}\left\{2 \rho_{k}^{2}\left(k^{2}+J\right)+\right. \\
& \sum_{n, m}\left[u_{n m}(k) \cos \left(n \beta \phi_{1}\right) \cos \left(m \beta \phi_{2}\right) J_{0}\left(2 n \beta \rho_{k}\right) J_{0}\left(2 m \beta \rho_{k}\right)\right. \\
& \left.\left.+v_{n m}(k) \sin \left(n \beta \phi_{1}\right) \sin \left(m \beta \phi_{2}\right) J_{0}\left(2 n \beta \rho_{k}\right) J_{0}\left(2 m \beta \rho_{k}\right)\right]\right\},
\end{aligned}
$$

where $J_{0}(x)$ stands for the Bessel function.

Eqs. (17) and (19) can be used for the determination of the RG flow of the generalized LSG model in the LPA, but they are solvable only numerically. Instead, we give below an analytic solution of the linearized RG equations which is valid only in the UV scaling regime. Nevertheless, it has the value of being closely related to the result obtained in the dilute gas approximation (Pierson and Valls 1992, Pierson et. al. 1992) and with its help one can recognize the improvement to be expected form the numerical determination of the WH-RG flow in the LPA.

\section{LINEARIZED RG}

In order to discuss the linearized RG flow of the generalized LSG model, the dimensionless WH-RG equation (11) obtained in LPA is linearized around the Gaussian fixed point

$$
\left(2+k \partial_{k}\right) \tilde{V}_{k}\left(\phi_{1}, \phi_{2}\right)=-\alpha_{2}\left(\tilde{V}_{k}^{11}+\tilde{V}_{k}^{22}\right)
$$


by assuming $1>>\left|\tilde{V}_{k}^{i j}\right|$ where $\tilde{V}_{k}^{i j}=\partial_{\phi_{i}} \partial_{\phi_{j}} \tilde{V}_{k}\left(\phi_{1}, \phi_{2}\right)$. Using the ansatz (14) and (15) for the generalized LSG model, the derivatives of the potential are

$$
\begin{aligned}
& \tilde{V}_{k}^{11}=\tilde{J}_{k}-\sum_{n, m} n^{2} \beta^{2}\left[\tilde{u}_{n m}(k) \cos \left(n \beta \phi_{1}\right) \cos \left(m \beta \phi_{2}\right)+\tilde{v}_{n m}(k) \sin \left(n \beta \phi_{1}\right) \sin \left(m \beta \phi_{2}\right)\right], \\
& \tilde{V}_{k}^{22}=\tilde{J}_{k}-\sum_{n, m} m^{2} \beta^{2}\left[\tilde{u}_{n m}(k) \cos \left(n \beta \phi_{1}\right) \cos \left(m \beta \phi_{2}\right)+\tilde{v}_{n m}(k) \sin \left(n \beta \phi_{1}\right) \sin \left(m \beta \phi_{2}\right)\right] .
\end{aligned}
$$

Inserting Eqs. (21) into the linearized WH-RG Eq. (20) the following RG equations are obtained for the coupling constants of the generalized LSG model

$$
\begin{aligned}
\left(2+k \partial_{k}\right) \tilde{J}_{k} & =0, \\
\left(2+k \partial_{k}\right) \tilde{u}_{n m}(k) & =\alpha_{2} \beta^{2}\left(n^{2}+m^{2}\right) \tilde{u}_{n m}(k), \\
\left(2+k \partial_{k}\right) \tilde{v}_{n m}(k) & =\alpha_{2} \beta^{2}\left(n^{2}+m^{2}\right) \tilde{v}_{n m}(k),
\end{aligned}
$$

where $\alpha_{2}=1 / 4 \pi$. The linearized RG equations for the various coupling constants decouple and their solutions can be obtained analytically

$$
\begin{gathered}
\tilde{J}_{k}=J_{k=\Lambda}\left(\frac{k}{\Lambda}\right)^{-2}, \\
\tilde{u}_{n m}(k)=\tilde{u}_{n m}(k=\Lambda)\left(\frac{k}{\Lambda}\right)^{-2+\alpha_{2} \beta^{2}\left(n^{2}+m^{2}\right)}, \\
\tilde{v}_{n m}(k)=\tilde{v}_{n m}(k=\Lambda)\left(\frac{k}{\Lambda}\right)^{-2+\alpha_{2} \beta^{2}\left(n^{2}+m^{2}\right)},
\end{gathered}
$$

where $J_{k=\Lambda}, \tilde{u}_{n m}(k=\Lambda)$ and $\tilde{v}_{n m}(k=\Lambda)$ are the initial values for the coupling constants at the UV cutoff $\Lambda$. The linearized RG flow predicts two phases of the model. If the parameter $\beta^{2}$ is larger than a critical value $\beta^{2}>\beta_{c}^{2}=8 \pi$, all the Fourier amplitudes $\tilde{u}_{n m}(k)$ and $\tilde{v}_{n m}(k)$ are irrelevant, they decrease when the cutoff $k$ is moved towards zero. For $\beta^{2}<8 \pi$, at least one of the Fourier amplitudes becomes relevant. This phase structure is very similar to that obtained for the massless SG model (Nandori et. al. 2001, 2004). Nevertheless, there is a significant difference. In case of the generalized LSG model, the coupling constant $\tilde{J}_{k}$ is always a relevant parameter and, consequently, the linearization loses its validity with decreasing scale $k$ for any value of $\beta$. Therefore, one has to determine the WH-RG flow with taking all the non-linear terms into account in order to obtain reliable IR behaviour for the coupling constants. The numerical solution of the problem is in progress.

Finally, the UV scaling laws obtained above and applied to the LSG model (3) via

$$
\tilde{u}_{10}(k)=\tilde{u}_{01}(k)=\tilde{u}(k),
$$


are compared to the flow given by Eq. (44) found in Ref. (Pierson and Valls 1992, Pierson et. al. 1992) in the dilute gas approximation. The linearized WH-RG equations (22) applied to the LSG model reduce to

$$
k \frac{d}{d k} \beta^{2}=0, \quad k \frac{d}{d k} \tilde{u}=\tilde{u}\left(\frac{\beta^{2}}{4 \pi}-2\right), \quad k \frac{d}{d k} \tilde{J}=-2 \tilde{J} .
$$

These turn out to be the same as those of Eq. (4) found in Ref. (Pierson and Valls 1992) except of the loss of the scale-dependence of $\beta$ due to the usage of the LPA. Therefore, our approach with the non-linear terms included in the RG equations offers the possibility to achieve an improvement in the determination of RG flow as compared to the results of the dilute gas approximation.

\section{SUMMARY}

The new qualitative picture of the vortex length-scale dependence obtained by recent current transport measurements on BSCCO high temperature superconductor (HTSC) single crystals indicates the need for a better description of the critical behaviour of vortices in layered systems. In this paper we investigated the phase structure and the critical behaviour of the layered sine-Gordon (LSG) model which is assumed to be one of the models describing the vortex dominated properties of HTSC materials reasonably well. The renormalization of the LSG model has been considered by the differential renormalization group (RG) method using a sharp momentum cutoff. The usage of sharp cutoff is a necessity if a spinodal instability occurs during the renormalization of the model. The Wegner-Houghton (WH) RG equation as well as the tree-level blocking relation (which one should use in case of spinodal instability) have been derived in the local potential approximation for the LSG model. The UV scaling laws and the phase structure of the LSG model have been discussed at the linearized level by the WH-RG method. The well-known UV scaling laws obtained in the dilute gas approximation previously in the literature (Pierson and Valls 1992, Pierson et. al. 1992) were recovered. Finally, it was argued that our approach offers an improvement in the determination of the RG flow as compared to that found in the dilute gas approximation when all the non-linear terms are taken into account in the WH-RG equation. 


\section{Acknowledgments}

I. N. thanks the Max-Planck-Institute for Nuclear Physics, Heidelberg, for the kind hospitality extended on the occasion of a guest researcher appointment in 2004 during which part of this work was completed. I.N. also takes a great pleasure in acknowledging discussion with K. Vad, S. Mészáros and U.D. Jentschura. This work has been supported by the grant OTKA T032501/00 and also partially by the grant OTKA M041537 and the Supercomputing Laboratory of the Faculty of Natural Sciences, University of Debrecen. 
ALEXANDRE J., POLONYI J., 1999, Phys. Lett. B. 445351.

NÁNDORI I., POLONYI J., SAILER K., 2001, Phys. Rev. D. 63 045022.; 2001, Phil. Mag. B. 81 1615.

NÁNDORI I., SAILER K., JENTSCHURA U. D., SOFF G., 2004, Phys. Rev. D. 69 025004.; 2002, J. Phys. G. 28607.

NÁNDORI I., VAD K., MÉSZÁROS S., HAKL J., SAS B., 2004, Czech. J. Phys. 54 D481.

PIERSON S. W., 1995, Phys. Rev. Lett. 74, 2359.; 1997, Phys. Rev. B 55, 14536.

PIERSON S.W., Valls O. T., 1994, Phys. Rev. B 49, 662.

PIERSON S.W., Valls O. T., 1992, Phys. Rev. B 45, 13076.

PIERSON S.W., Valls O. T., BAHLOULI H., 1992, Phys. Rev. B 45, 13035.

LIAO S. B., POLONYI J., 1995, Phys. Rev. D. 514474.

POLONYI J., 2001, Lectures on Functional Renormalization Group Method, hep-th/0110026.

PORTIER F., et. al. 2002, Phys. Rev. B 66, 511.

WEGner F. J., HOUGHTON A., 1973, Phys. Rev. A. 8401.

WILSON K. G., KOGUT J., 1974, Phys. Rep. C. 12 77;

WILSON K. G., 1975, Rev. Mod. Phys. 47 773; 1983, Rev. Mod. Phys. 55583. 